\title{
Fabrication and Characterization of Magnesium Nanoparticle by Gas Evaporation Method*
}

\author{
S. Ogawa ${ }^{\dagger}$ and H. Niwa \\ Department of Quantum Engineering, School of Engineering, \\ Nagoya University, Furo-cho, Chikusa-ku, Nagoya 464-8603, Japan \\ T. Nomoto \\ Aichi Industrial Technology Institute, Onda-cho 1-15\%-1, Kariya-city 448-0013, Japan \\ S. Yagi \\ Department of Quantum Engineering, School of Engineering, \\ Nagoya University, Furo-cho, Chikusa-ku, Nagoya 464-8603, Japan. \\ (Received 10 January 2010; Accepted 18 March 2010; Published 15 May 2010)
}

\begin{abstract}
We have fabricated the $\mathrm{Mg}$ nanoparticles by the gas evaporation method. The AFM observation shows $\mathrm{Mg}$ nanoparticles fabricated under the several gas pressures of He have the average diameter of $3.6 \mathrm{~nm}$ to $8.0 \mathrm{~nm}$. We have studied the influence of the atmospheric oxidation for the chemical state of the $\mathrm{Mg}$ nanoparticles by the $\mathrm{Mg}$ K-edge NEXAFS technique. The NEXAFS measurement shows that the chemical state of $\mathrm{MgCO}_{3}$ exists on the most lateral surface of the $\mathrm{Mg}$ nanoparticles and the degree of atmospheric oxidation depends on the size of $\mathrm{Mg}$ nanoparticles. [DOI: 10.1380/ejssnt.2010.246]
\end{abstract}

Keywords: Mg nanoparticle; Gas evaporation method; Atmospheric oxidation; AFM; NEXAFS

\section{INTRODUCTION}

The main energy resources are fossil fuel such as the oil, and the usage increases year by year. However, the environmental burden by using of large quantity of fossil fuel becomes very serious problem. Therefore, sustainable energy resources are required. The hydrogen is paid attention as the clean energy resources because the hydrogen generates only water when it reacts with the oxygen [1]. For use of the hydrogen as the fuel, we need to establish its effective storage technique.

Hydrogen storage material, which storages the hydrogen inside and releases it by heating, is required lightweight and inexpensive material compared with the hydrogen gas chamber [2]. The alloys based on $\mathrm{Mg}$ are more lightweight and inexpensive materials than other materials, and $\mathrm{Mg}$ possesses of $7.6 \mathrm{wt} \%$ storage for hydrogen [3]. $\mathrm{Mg}$ absorbs and desorbs hydrogen at high temperature range of $623-673 \mathrm{~K}$ [3], but MgNi alloy film coated with $\mathrm{Pd}$ can absorb and desorb hydrogen at room temperature and under atmospheric pressure [4].

Our idea to increase the hydrogen storage capacity of this film is increase of the surface area for reaction with hydrogen, in other words, fabrication of the film consists of nanoparticles. It is difficult to fabricate alloy nanoparticles because of difference of melting point of each component elements. Therefore, firstly we try to fabricate single component nanoparticles of $\mathrm{Pd}, \mathrm{Ni}$ and $\mathrm{Mg}$. After that, we try to fabricate the alloy nanoparticles by means of mixing three kinds of single nanoparticles. We have

*This paper was presented at 7th International Symposium on Atomic Level Characterizations for New Materials and Devices, The Westin Maui Resort \& Spa, Hawaii, U.S.A., 6-11 December, 2009. †Corresponding author: ogawa.satoshi@g.mbox.nagoya-u.ac.jp already fabricated $\mathrm{Pd}$ and $\mathrm{Ni}$ nanoparticles [5]. In this study we have tried to fabricate $\mathrm{Mg}$ nanoparticles.

We pay attention to size dependence of hydrogen storage capacity of $\mathrm{Mg}$ nanoparticles. In the hydrogen storage of $\mathrm{Mg}$, magnesium hydride $\left(\mathrm{MgH}_{2}\right)$ forms on the $\mathrm{Mg}$ surface firstly, and magnesium hydride layer prevents hydrogen atoms from penetrating to materials. Because the small nanoparticles have a larger specific surface area than the large ones, it is expected that the small $\mathrm{Mg}$ nanoparticles have high hydrogen storage capacity per its volume. For this reason, it is necessary to control the size of $\mathrm{Mg}$ nanoparticles for the fabrication of small $\mathrm{Mg}$ nanoparticles (about 1 or $2 \mathrm{~nm}$ ) and to compare with the small $\mathrm{Mg}$ nanoparticles and large ones about hydrogen storage capacity.

It is known that $\mathrm{Mg}$ loses hydrogen storage ability by the atmospheric oxidation [3]. Mg surface is oxidized easily by exposure to atmosphere, and $\mathrm{MgO}$ layer produced by atmospheric oxidation prevents hydrogen molecules from penetrating into the material. By a long time exposure to atmosphere for about 20 days, magnesium carbonates are formed on the $\mathrm{Mg}$ lateral surface, and oxides and hydroxides are produced under the magnesium carbonates layer [6]. In the case of the highly humid air, hydroxy carbonates $\left(\mathrm{MgCO}_{3} \cdot \mathrm{Mg}(\mathrm{OH})_{2} \cdot 3 \mathrm{H}_{2} \mathrm{O}\right)$ are formed [7].

In this study, we have fabricated the $\mathrm{Mg}$ nanoparticles and investigated the chemical state of $\mathrm{Mg}$ nanoparticles surface as a first step. The size of $\mathrm{Mg}$ nanoparticles is estimated by atomic force microscopy (AFM) observation and surface chemical state is studied by near-edge X-ray absorption fine structure (NEXAFS) measurement.

\section{EXPERIMENTAL}

The Mg nanoparticles were fabricated by the gas evaporation method [5]. $\mathrm{Mg}$ was evaporated at several $\mathrm{kPa}$ 

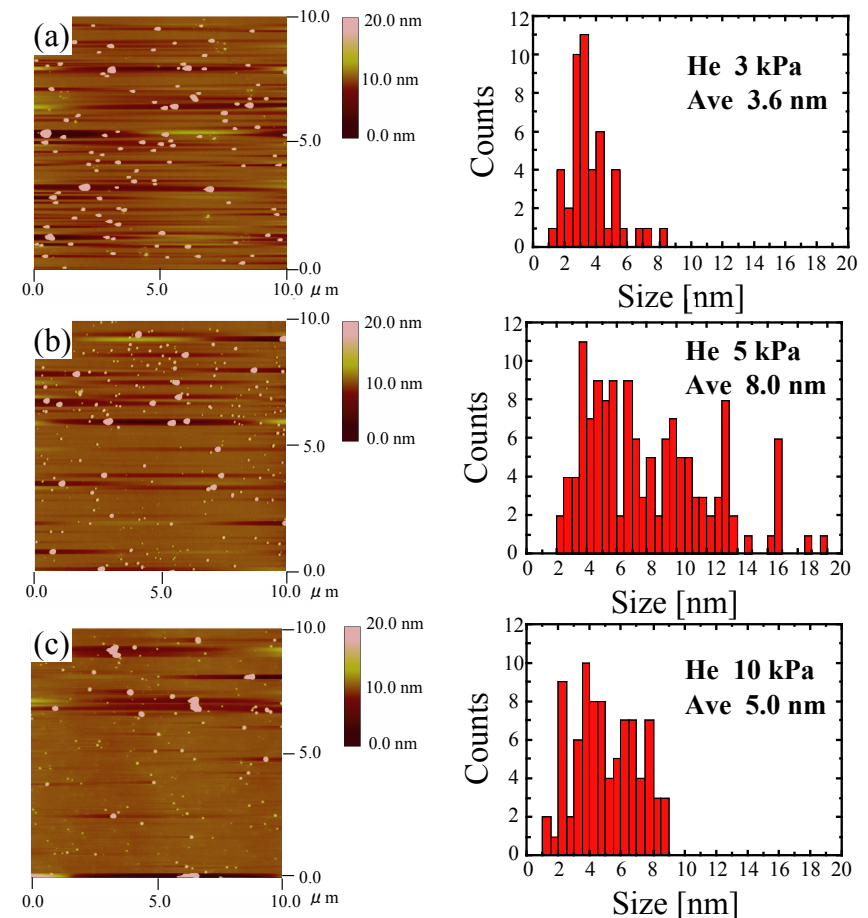

FIG. 1: AFM images and size distributions of Mg nanoparticles deposited on Si wafer.

of He gas. $\mathrm{Mg}$ atoms are cooled down by He atoms and form nanoparticles by aggregation each other. The $\mathrm{Mg}$ nanoparticles were deposited on the Si substrate and the amount was controlled by the deposition time. $\mathrm{Mg}$ thin film (100 nm thickness) on $\mathrm{Ni}$ was fabricated by magnetron sputtering as the standard sample of $\mathrm{Mg}$ bulk.

The AFM observations were carried out under atmospheric condition by using Nanoscope IIIa (Veeco) by tapping mode. The Mg K-edge NEXAFS measurements were done at BL-10 of the SR center in Ritsumeikan University by total electron yield (TEY) method with sample drain current and fluorescence X-ray yield (FY) method with silicon drift detector. All NEXAFS spectra were obtained under high vacuum condition.

\section{RESULTS AND DISSCUSSION}

\section{A. AFM images}

Figures 1(a), (b), and (c) show the AFM images of the $\mathrm{Mg}$ nanoparticles on Si substrate fabricated at 3, 5 and $10 \mathrm{kPa}$ of $\mathrm{He}$, respectively. Since the height value of the AFM analysis has a good resolution in comparison with the horizontal one, the diameter of nanoparticles is evaluated by the height value. Judging from the results of AFM observations, it is found that the $\mathrm{Mg}$ nanoparticles fabricated at 3,5 and $10 \mathrm{kPa}$ have the average diameter of 3.6 , 8.0 and $5.0 \mathrm{~nm}$, respectively. Yagi et al. have reported that $\mathrm{Pd}$ nanoparticles fabricated by the gas evaporation method have the diameter proportional to He gas pressure [5]. However, such tendency is not seen for the $\mathrm{Mg}$ nanoparticles in this study.

The melting point of $\mathrm{Mg}(923 \mathrm{~K})$ is much lower than

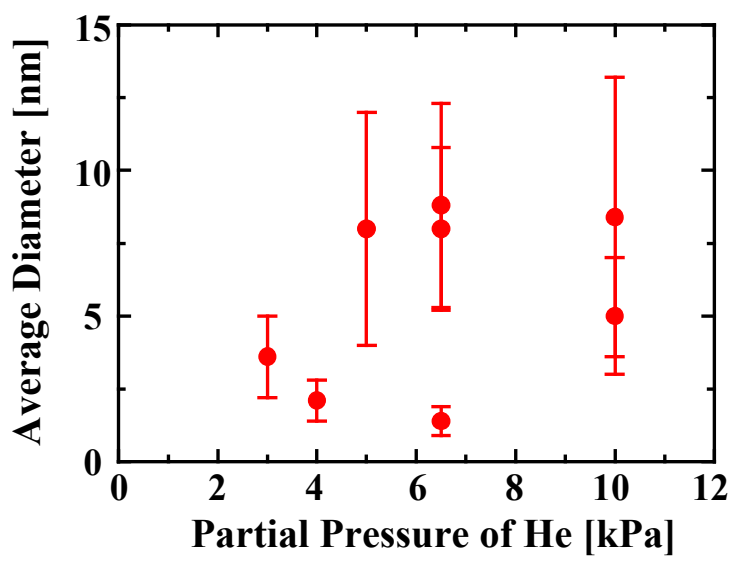

FIG. 2: The relation between partial pressure of He and average diameter of $\mathrm{Mg}$ nanoparticles.

that of $\mathrm{Pd}(1828 \mathrm{~K})$. Moreover, the vapor pressure of $\mathrm{Mg}$ at melting point is much higher than that of $\mathrm{Pd}$. Both the low melting point and the high vapor pressure increase the evaporation rate of $\mathrm{Mg}$ source, and it causes much aggregation of $\mathrm{Mg}$ atoms. It is thought that the control of the size of $\mathrm{Mg}$ nanoparticles is difficult because the high evaporation rate of $\mathrm{Mg}$ is much more significant factor than the He gas pressure in the form of $\mathrm{Mg}$ nanoparticles.

In addition, we evaporate $\mathrm{Mg}$ source by the resistive heating of the tungsten (W) basket, and the setting position of $\mathrm{Mg}$ source on $\mathrm{W}$ basket is slightly different a little at each fabrication of $\mathrm{Mg}$ nanoparticles. A little difference of the setting position of source influences the evaporation rate significantly. Thereby, shown in Fig. 2, the repeatability of the size of $\mathrm{Mg}$ nanoparticles which fabricated under each partial pressures of He is not obtained.

\section{B. Mg K-edge NEXAFS}

Figures 3(A) and (B) show the Mg K-edge NEXAFS spectra for the $\mathrm{Mg}$ nanoparticles oxidized in atmosphere for about 20 days. The spectra in (A) and (B) have been taken by FY and TEY methods, respectively. As a standard sample, we have measured $\mathrm{Mg}$ bulk, $\mathrm{MgO}$ and magnesium hydroxy carbonates $\left(4 \mathrm{MgCO}_{3} \cdot \mathrm{Mg}(\mathrm{OH})_{2} \cdot 4 \mathrm{H}_{2} \mathrm{O}\right)$.

In the (A)-(a) 1 spectrum for small amount of deposition the peak associated with the chemical state of $\mathrm{MgCO}_{3}$ [8] can be seen clearly, but in the (A)-(a) 2 spectrum for large amount of deposition the peak of $\mathrm{MgCO}_{3}$ is not seen clearly and the peak of $\mathrm{MgO}$ is significant. In the (A)-(b) spectrum the peak associated with the chemical state of $\mathrm{Mg}$ bulk can be observed. Since the peak top has a flat shape, it seems that both $\mathrm{MgO}$ and $\mathrm{MgCO}_{3}$ compounds exist.

In the (B)-(a)1 spectrum the peak of $\mathrm{MgCO}_{3}$ is seen clearly and the shoulder structure of $\mathrm{MgO}$ is also observed. The peak intensity of (B)-(a)2 spectrum becomes small because the peak of $\mathrm{MgO}$ is weakened by self absorption effect in fluorescence $\mathrm{x}$-ray absorption. The shape of the (B)-(b) spectrum is almost similar with the (A)-(b) spectrum, but intensity of the peak of $\mathrm{Mg}$ bulk is higher than that of the (A)-(b) spectrum. 

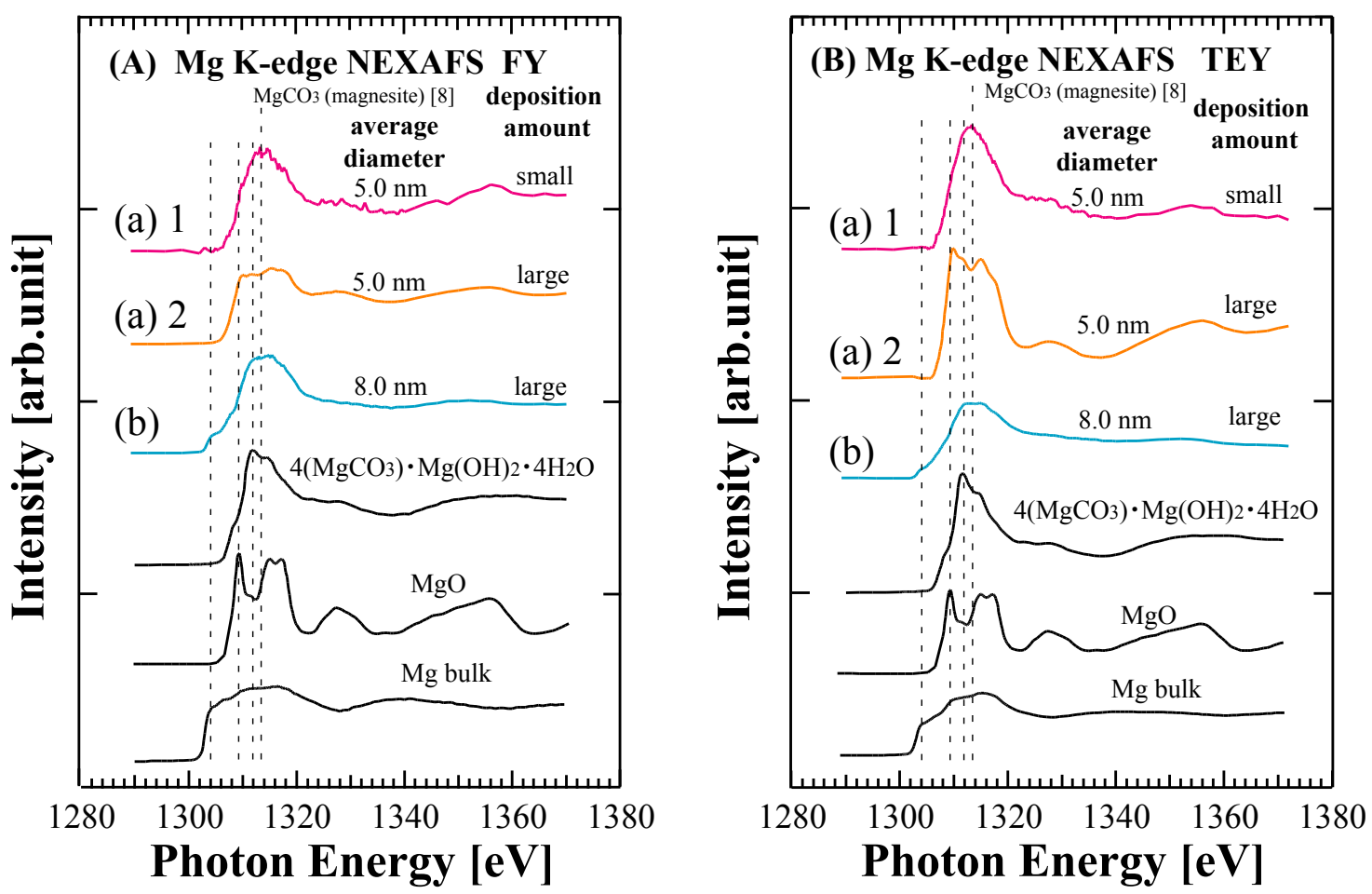

FIG. 3: Mg K-edge NEXAFS spectra for Mg nanoparticles measured by (A) TEY and (B) FY methods. All samples have been exposed to atmosphere for about 20 days.

These results mean that $\mathrm{MgO}$ and $\mathrm{MgCO}_{3}$ exist on the surface of the $\mathrm{Mg}$ nanoparticles. Consider the deposition amount of $\mathrm{Mg}$ nanoparticles, it is thought that the adsorbate at lateral surface on $\mathrm{Mg}$ nanoparticles is dominantly $\mathrm{MgCO}_{3}$. The $\mathrm{Mg}$ nanoparticles with average diameter of $5 \mathrm{~nm}$ are fully oxidized by atmosphere. On the other hand, metal $\mathrm{Mg}$ atoms exist inside of the $\mathrm{Mg}$ nanoparticle with average diameter of $8 \mathrm{~nm}$.

Judging from the intensity of the shoulder structure associated with the chemical state of $\mathrm{Mg}$ bulk in NEXAFS spectra, following things can be said. The small nanoparticles have very large specific surface area, thereby the inside of the small nanoparticles are easily oxidized by atmosphere. With increase the size of nanoparticle, the specific surface area decreases and bulk component increases gradually. Therefore, it is thought that the large nanoparticles are difficult to progress atmospheric oxidation in comparison with the small nanoparticles.

We have speculated that the reaction time for the atmospheric oxidation of $\mathrm{Mg}$ nanoparticles becomes short because of the large specific surface area. However, it is revealed by the NEXAFS measurement that the atmospheric oxidation of $\mathrm{Mg}$ nanoparticles has not completed for about 20 days, and $\mathrm{MgO}$ and $\mathrm{MgCO}_{3}$ have formed on the surface of $\mathrm{Mg}$ nanoparticles. We are going to clarify the variation of those products of the atmospheric oxidation for a long reaction time, for example several months, and the influence on hydrogen storage ability.

\section{CONCLUSION}

We have fabricated the $\mathrm{Mg}$ nanoparticles by the gas evaporation method, but the size control has not been established. The chemical state of $\mathrm{Mg}$ nanoparticles has been characterized by Mg K-edge NEXAFS technique. It is found that the chemical state of $\mathrm{MgCO}_{3}$ exists on the lateral surface of the $\mathrm{Mg}$ nanoparticles. By the exposure to atmosphere for 20 days, $\mathrm{Mg}$ nanoparticles with average diameter of $5 \mathrm{~nm}$ are fully oxidized, but metal $\mathrm{Mg}$ atoms exist inside of $\mathrm{Mg}$ nanoparticles with average diameter of $8 \mathrm{~nm}$.

\section{Acknowledgments}

The authors are grateful for the financial supported of a Grant-in-Aid for Scientific Research from the Ministry of Education, Science and Culture, Japan (No. 19008872) and Knowledge Cluster II in Aichi/Nagoya area. This work was performed under the approval of Ritsumeikan University SR center Program Advisory Committee (No. S21-06). Especially NEXAFS measurements are supported by the nanotechnology network project of the Ministry Education, Culture, Sports, Science and Technology, JAPAN (MEXT).
[1] S. Dunn, Int. J. Hydrogen Energy 27, 235 (2002).

[2] A. Züttel, Materials Today 6, 24 (2003).
[3] A. Zaluska, L. Zaluski, and J. O. Strom-Olsen, J. Alloys Compd. 288, 217 (1999). 
[4] K. Yoshimura, J. Surf. Finishing Soc. Jpn. 56, 882 (2005).

[5] S. Yagi, H. Sumida, K. Miura, T. Nmoto, K. Soda, G. Kutluk, H. Namatame, and M. Taniguchi, e-J. Surf. Sci. Nanotech. 4, 258 (2006).

[6] C. Fotea, J. Callaway, M R. Alexander, Surf. Interface Anal. 38, 1363 (2006).
[7] R. Lindstrom, L.-G. Johansson, G. E. Thompson, P. Skeldon, and J.-E. Svensson, Corr. Sci. 38, 1363 (2006).

[8] F. Farges, M.-P. Etcheverry, A. Haddi, P. Trocellier, E. Curti, and G. E. Brown Jr., XAFS 13 (2007). 